Presented at: Micro Bunches Workshop

Upton, NY

September 28-30, 1995

CONF-950922 q 16

BNL- 63987

Storage Ring Working Group Report

\author{
S. Krinsky \\ National Synchrotron Light Source \\ Brookhaven National Laboratory \\ Upton, NY 11973-5000
}

9506842

January 1997

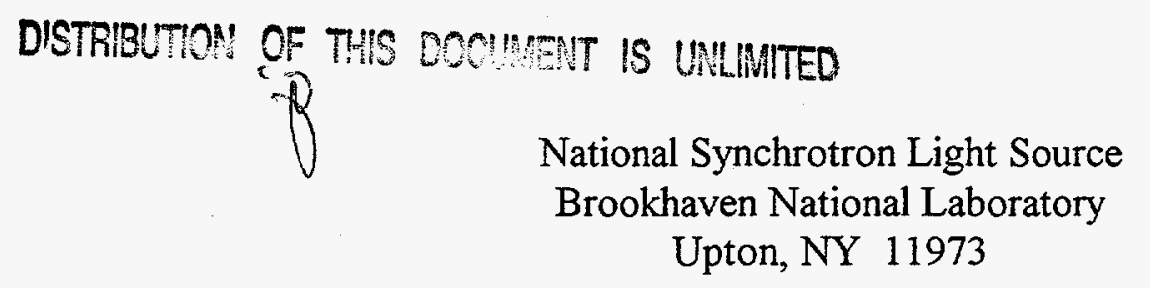

MASTER

Work performed under the auspices of the U.S. Department of Energy, under contract DE-AC02-76CH00016 


\section{DISCLAIMER}

Portions of this document may be illegible in electronic image products. Images are produced from the best available original document. 


\section{DISCLAIMER}

This report was prepared as an account of work sponsored by an agency of the United States Government. Neither the United States Government nor any agency thereof, nor any of their employees, make any warranty, express or implied, or assumes any legal liability or responsibility for the accuracy, completeness, or usefulness of any information, apparatus, product, or process disclosed, or represents that its use would not infringe privately owned rights. Reference herein to any specific commercial product, process, or service by trade name, trademark, manufacturer, or otherwise does not necessarily constitute or imply its endorsement, recommendation, or favoring by the United States Government or any agency thereof. The views and opinions of authors expressed herein do not necessarily state or reflect those of the United States Government or any agency thereof. 


\title{
Storage Ring Working Group Report
}

\author{
S. Krinsky \\ National Synchrotron Light Source \\ Brookhaven National Laboratory, Upton, New York 11973
}

\section{INTRODUCTION}

Over the last two decades great progress has been made in the development of storage rings with small transverse emittance. It is now a good time to consider the possibility of achieving very short bunches in storage rings. From the perspective of synchrotron radiation source development, there are at least two motivations for obtaining short electron bunches: (1) the generation of sub-picosecond $x$-ray pulses and (2) the coherent emission of sub-picosecond pulses of far infrared radiation.

A useful short-term goal is the experimental study of bunches with 1-ps rms length, both at high ( $21 \mathrm{GeV})$ and low $(\leqslant 150 \mathrm{MeV})$ electron energies. Experiments on 1-ps bunches are now feasible and can yield new insight into the high frequency impedance of storage rings and the associated phenomena which can result in bunch lengthening. Achievement of 1-ps bunches can also be expected to allow the first observation of coherent synchrotron radiation in a storage ring, in the millimeter wavelength regime.

A longer-term objective is the realization of $100-\mathrm{fs}$ bunches. Achievement of this goal not only will advance understanding of storage rings but will open up new opportunities in synchrotron radiation based research at both $x$-ray and far infrared wavelengths. It is now an appropriate time to carry forward theoretical investigations clarifying the fundamental limitations on bunch length, and to devise schemes to minimize it.

\section{EXPERIMENTS}

In the working group there was discussion of possible experiments aimed at achieving short bunches in storage rings.

\section{ESRF at $1 \mathrm{GeV}$}

In these proceedings [1] it is reported that by operating the ESRF storage ring at low energy ( $1 \mathrm{GeV}$ rather than $6 \mathrm{GeV}$ ) and high $\mathrm{RF}$ overvoltage, bunches of 1-ps 
length have been achieved. Work was confined to one machine-studies period so results were not optimized. It is estimated that there were about $10^{7}$ electrons/bunch. Coherent synchrotron radiation can be observed at wavelengths in the interval [2]

$$
\pi \sigma_{s} \leq \lambda \leq 2 h \sqrt{\frac{h}{\rho}},
$$

where $\sigma_{s}$ is the rms bunch length, $h$ is the full vertical aperture of the chamber, and $\rho$ is the bending radius. Taking $\sigma_{s}=0.3 \mathrm{~mm}, h=30 \mathrm{~mm}$ and $\rho=25 \mathrm{~m}$, one finds that coherent synchrotron radiation at ESRF should be observable in the range

$$
1 \mathrm{~mm} \leq \lambda \leq 2 \mathrm{~mm} \text {. }
$$

By observing radiation from a superconducting wiggler with bending radius $\rho=6 \mathrm{~m}$, the interval is increased to

$$
1 \mathrm{~mm} \leq \lambda \leq 4 \mathrm{~mm} .
$$

\section{Low Energy, Small Bending Radius, High Voltage}

Experiments complementary to the work at ESRF could be carried out on a low energy storage ring $(\leqslant 150 \mathrm{MeV})$ with small bending radius $(\leqslant 0.5 \mathrm{~m})$ and high RF frequency and voltage. Two such experiments $[3,4]$ were discussed at the workshop. In these experiments coherent synchrotron radiation will propagate in the larger wavelength interval

$$
1 \mathrm{~mm} \leqslant \lambda \leqslant 15 \mathrm{~mm} .
$$

The emission of synchrotron radiation results in an impedance for the electron beam, which for these small storage rings may be the dominant impedance.

\section{Low Momentum Compaction $\alpha$}

Several groups [5] are working to reduce the bunch length in existing storage rings by working near $\eta=\alpha-1 / \gamma^{2} \approx 0$. In order to maintain a large enough energy acceptance to have a long lifetime, it is necessary to reduce the second-order momentum compaction $\alpha_{2}$ by adjusting sextupole strengths. Experiments with this objective are on-going. At the present time bunch lengths on the order of $10 \mathrm{ps}$ have been achieved. 


\section{Synchrotron Tune $v_{\mathrm{s}} \approx 0.5$.}

Billings [6] has pointed out that to achieve very short bunches it may be advantageous to work near synchrotron tune $v_{s} \approx 0.5$. In this case the bunch length will vary strongly around the storage ring, being minimized at a certain location in analogy to focusing the beam transversely in a low- $\beta$ insertion. One must introduce a longitudinal beta function to describe the azimuthal variation of the bunch length.

The RF cavities which give a change in energy proportional to the RF phase of an electron are focusing elements (analogous to quadrupoles in transverse optics). The path length traversed by an electron in a bending magnet (and hence its change in RF phase) is proportional to the electron energy. Therefore, the bending magnets are drift elements. One advantage of having very short bunch length in only a small portion of the ring is that lifetime and instability effects associated with short bunch length might be reduced.

\section{Local Bunch Compression}

John Byrd brought to the attention of the working group an idea introduced by Orlov [7] to carry out local bunch compression. This approach is based on the use of an RF cavity operating in a transverse deflecting mode, in order to deliver a transverse kick resulting in a transverse angular deflection $\Delta x^{\prime}(s)$ that depends on $s$, the longitudinal coordinate of the electrons within the bunch. The particles then traverse a segment of the ring lattice, containing bending and quadrupole magnets, having the property that electrons with different $s$ move along different trajectories and are focused longitudinally.

\section{Intrabunch Microbunching}

Unless one uses an isochronous storage ring with very small momentum compaction, the microbunching produced during one pass through an FEL can be expected to be randomized before its re-entry after one revolution. However, it has recently been suggested [8] that there may be a way to overcome this difficulty by operating near a high-order nonlinear synchrotron oscillation (and possibly betatron oscillation) resonance of the storage ring. In this case the phase space of the bunch can be expected to repeat after a finite number (e.g. 10) of revolutions around the ring. This may allow for maintaining a high degree of microbunching. 


\section{THEORY}

In an electron storage ring, when we ignore wakefield effects, the distribution function $\mathrm{f}(\phi, \epsilon, t)$ in longitudinal phase $\phi$ and energy $\epsilon$ satisfies a Fokker-Planck equation of the form [9]

$$
\frac{\partial \mathrm{f}}{\partial t}+\epsilon \frac{\partial \mathrm{f}}{\partial \phi}-\left(\frac{d V}{d \phi}+\frac{\epsilon}{\tau}\right) \frac{\partial \mathrm{f}}{\partial \epsilon}-\frac{1}{\tau} \mathrm{f}-D \frac{\partial^{2} \mathrm{f}}{\partial \epsilon^{2}}=0 .
$$

Here, $\tau$ is the radiation damping time, $D$ is the diffusion constant resulting from quantum fluctuations, and $V(\phi)$ is the RF potential. It was shown by Hereward [9] that the time-independent solution of this equation is

$$
f(\phi, \epsilon)=A e^{-\frac{1}{\sigma^{2}}\left[\frac{\epsilon^{2}}{2}+V(\phi)\right]},
$$

where $\mathrm{A}$ is a normalization constant and

$$
\sigma=\sqrt{\mathrm{D} \tau} .
$$

Wakefield effects can be considered by taking

$$
\frac{d \mathrm{~V}}{d \phi}=\phi+\int_{-\infty}^{\infty} d \phi^{\prime} G\left(\phi-\phi^{\prime}\right) \lambda\left(\phi^{\prime}\right),
$$

where the RF potential has been assumed to be harmonic, and the wakefield is described by the Green's function $G\left(\phi-\phi^{\prime}\right)$. The line charge density $\lambda(\phi)$ is

$$
\lambda(\phi)=\int d \epsilon \mathrm{f}(\phi, \epsilon) .
$$

Haissinki [10] found the time-independent solution

$$
f(\phi, \epsilon)=e^{-\epsilon^{2} / 2 \sigma^{2}} g(\phi),
$$

where $g(\phi)$ is determined by 


$$
\frac{d g}{d \phi}+\frac{\phi g(\phi)}{\sigma^{2}}+\frac{g(\phi)}{\sigma^{2}} \int_{-\infty}^{\infty} d \phi^{\prime} G\left(\phi-\phi^{\prime}\right) g\left(\phi^{\prime}\right)=0
$$

Alternatively, one can write

$$
g(\phi)=C \mathrm{e}^{-\frac{\phi^{2}}{2 \sigma^{2}}-\frac{1}{\sigma^{2}} \int_{0}^{\phi} d \phi^{\prime} \int_{-\infty}^{\infty} d \phi^{\prime \prime} G\left(\phi^{\prime}-\phi^{\prime \prime}\right) g\left(\phi^{\prime \prime}\right)},
$$

the constant $C$ being determined by the normalization condition on the distribution, and $\sigma$ again being given by Eq. (7).

Suppose there exists a solution of the Haissinki, Eq.(12). The stability of this solution can be checked by carrying out a linear analysis of the Fokker-Planck Eq. (5) [ with $d V / d \phi$ given by Eq. (8)]. One writes

$$
\mathrm{f}(\phi, \epsilon, t)=\mathrm{e}^{-\epsilon^{2} / 2 \sigma^{2}} g(\phi)+f_{1}(\phi, \epsilon, t),
$$

and employs this expression in Eq. (5) keeping only terms linear in $f_{1}$. Such an analysis was carried out by Renieri [11].

For peak currents below the threshold for turbulent bunch lengthening the Haissinki solution is stable, and the effect of the wakefield is to modify the longitudinal bunch profile without changing the energy spread. Above the threshold, the Haissinki solution is unstable and the behavior is more complex, with modification of the energy and phase distributions ensuing. It has been suggested [12] that an "Haissinki-like" equation can be used to determine the equilibrium solution above threshold. One way to conceive of such a possibility is to consider the "microwave instability" to result in a new equilibrium described by a modified diffusion constant $\tilde{D}$. The increased energy spread $\tilde{\sigma}$ in this case would be determined by

$$
\tilde{\boldsymbol{\sigma}}=\sqrt{\tilde{D} \tau},
$$

and the new longitudinal profile $\tilde{g}(\phi)$ might be a solution of the "Haissinki-like" equation obtained from Eq. (12) by replacing $\sigma$ by $\widetilde{\sigma}$. This type of equilibrium was suggested by Sessler [13], and might be a justification for the more recent work of Bane and Ruth [14].

On the other hand it is clear that once the Haissinki solution is unstable, it is very possible that the bunch distribution will not come to a time-independent equilibrium. The "sawtooth" instability [15] observed in the SLC damping ring is an example of such time-dependent behavior. Above a current threshold, the bunch 
length is observed to undergo relaxation oscillations, periodically increasing to a larger value and then collapsing back to a smaller one.

\section{CONCLUDING REMARKS}

In this working group many approaches to the achievement of short bunches in storage rings were proposed, and much will be learned if experiments based on these ideas are carried out. Operation of a storage ring in the regime of coherent emission of far infrared radiation is of great interest, and may offer some surprises. The theoretical description of wakefield effects on the longitudinal energy and phase distribution deserves further attention. Although much work has been done on this problem, important open questions remain.

\section{REFERENCES}

1. L. Farvacque, T. Gunzel, J.L. Laclare, C. Limborg, A. Ropert and U. Weinrich, these proceedings.

2. See e.g., R.L. Warnock, "Shielded Coherent Synchrotron Radiation and Its Possible Effect in the Next Linear Collider," Proc. 1991 IEEE Part. Accel. Conf., San Francisco, CA (1991), 1824.

3. H. Yamada, these proceedings.

4. J.B. Murphy and S. Krinsky, Nucl. Instr. Meth. A346 (1994) 571.

5. D. Robin, H. Hama, and A. Nadji, these proceedings.

6. M. Billings, unpublished.

7. Y.F. Orlov, C.M. O'Neill, J.J. Welch, "B Factory Optics and Beam-Beam Interaction for Millimeter $\beta^{*}$ And Locally Shortened Bunches, " Proc. 1991 IEEE Part. Accel. Conf., San Francisco, CA (1991) 2838.

8. B. Burnham, V. N. Litvinenko, J.M.J. Maday, and Y. Wu, these proceedings.

9. H.G. Hereward, "Equilibrium Energy Distribution in a Non-Linear Potential Well in the Presence of Quantum Fluctuations," PEP Note 53 (1973).

10. J. Haissinki, Nuovo Cimento 18B (1973) 72.

11. A. Renieri, "Turbulence and Bunch Lengthening in Electron-Positron Storage Rings," LNF-76/11 (R) (1976).

12. G. Besnier, J.L. Laclare and C. Limborg, these procedings.

13. A.M. Sessler, "Strongly Turbulent Collective Motion and the Anomalous Size of Stored Particle Beams," PEP Note 28 (1973).

14. K.F. Bane and R.D. Ruth, "Bunch Lengthening Calculation for the SLC Damping Rings, " Proc. 1989 IEEE Part. Accel. Conf., Chicago, Il (1989) 789.

15. K.F. Bane and K. Oide, "Simulations of the Longitudinal Instability in the SLC Damping Rings," Proc. 1993 Part. Accel. Conf., Washington D.C. (1993) 3339. 